\title{
HIPERTENSIÓN ARTERIAL PULMONAR
}

\section{Riociguat en el tratamiento de los pacientes con hipertensión pulmonar secundaria a tromboembolismo crónico}

\author{
Riociguat in the treatment of patients with pulmonary \\ hypertension secondary to chronic thromboembolism
}

Edward CÁceres MÉndez, MD. ${ }^{(1)}$; Claudio Villaquirán T., MD. ${ }^{(2)}$

(1)Residente de Segundo año de Medicina Interna, Candidato MSc Epidemiología Clínica Pontificia Universidad Javeriana, Hospital Universitario San Ignacio, Bogotá, Colombia.

(2) Internista, Neumólogo, MSc Hipertensión pulmonar, Candidato MSc Epidemiología Clínica, Hospital Universitario San Ignacio, Pontificia Universidad Javeriana, Bogotá, Colombia.

Correspondencia: Claudio Villaquirán $\mathrm{T}$. Correo electrónico: claudiovillaquiran@ gmail.com

Recibido: 20-10-2013. Aceptado: 15-11-2013.

\section{Referencia}

Ghofrani HA, D'Armini AM, Grimminger F, Hoeper MM, Jansa P, Kim NH et al, for the CHEST-1 Study Group. Riociguat for the treatment of chronic thromboembolic pulmonary hypertension. N Engl J Med. 2013;369:319-329.

\section{Pregunta}

¿En pacientes con hipertensión pulmonar tromboembólica crónica (HPTEC) inoperable o persistente, la terapia con riociguat mejora la distancia caminada durante la prueba de marcha de 6 minutos luego de 16 semanas de tratamiento cuando se compara con placebo?

\section{Diseño}

El estudio CHEST-1 (Chronic Thromboembolic Pulmonary Hypertension Soluble Guanylate Cyclase-Stimulator Trial 1) publicado en New England Journal of Medicine el 25 de julio de 2013, fue un experimento fase 3 , múlti-céntrico, doble ciego, aleatorio, controlado con placebo, diseñado por el primer autor y un comité interino, en colaboración con Bayer Health Care como patrocinador, cuyo protocolo fue aprobado por el comité de ética de cada institución participante. El análisis estadístico estuvo a cargo de bioestadísticos contratados por Bayer y fue revisado por el primer autor. El manuscrito fue elaborado por el primer autor, con apoyo editorial por parte del patrocinador. El monitoreo de seguridad se llevó a cabo sin cegamientos, en intervalos regulares. Todos los autores tenían acceso a la información recolectada, así como a la interpretación y redacción del manuscrito.

\section{Cegamiento}

Pacientes y clínicos evaluadores.

\section{Periodo de seguimiento}

Cuatro meses (16 semanas).

\section{Lugar}

89 centros médicos en 26 países. 


\section{Pacientes}

Se incluyeron pacientes de 18 a 80 años de edad con HPTEC reconocida como técnicamente inoperable, o hipertensión pulmonar persistente o recurrente después de someterse a trombo-endarterectomía pulmonar. Entre los criterios de inclusión adicionales estaban una distancia en la prueba de marcha de 6 minutos entre 150 a 450 metros, resistencia vascular pulmonar (RVP) mayor de 300 dinas $/ \mathrm{seg} / \mathrm{cm}^{-5}$, y presión media de la arteria pulmonar (PAPm) de al menos $25 \mathrm{~mm} \mathrm{Hg}$. El diagnóstico de HPTEC se realizó mediante el uso de dos o más de los siguientes métodos imagenológicos: gammagrafía pulmonar de ventilación-perfusión, angiografía pulmonar, tomografía axial computarizada o angiografía por resonancia magnética.

Se excluyeron quienes habían recibido un antagonista del receptor de endotelina, análogos de prostaciclina, inhibidor de la fosfodiesterasa tipo 5 o agonistas del óxido nítrico en los tres meses previos al ingreso al estudio. Se obtuvo el consentimiento informado escrito de todos los participantes.

\section{Intervención}

Los pacientes que se consideraron potencialmente elegibles para la participación ingresaron a una fase de pre-tratamiento en donde se efectuaba una evaluación sistemática respecto a la operabilidad y los demás criterios de inclusión. Luego de esta fase, los pacientes elegibles se asignaron al azar en una proporción de 1:2 a recibir placebo o riociguat. La titulación de este último se hizo a partir de una dosis inicial de $1 \mathrm{mg}$ tres veces al día de acuerdo con la presión arterial sistólica sistémica y los signos o síntomas de hipotensión (dosis final entre $0,5 \mathrm{mg}$ a $2,5 \mathrm{mg}$ tres veces al día). Las dosis alcanzada al final de la fase de titulación de 8 semanas se consideró como la dosis apropiada para el paciente, la cual se continuaba hasta el final del estudio. Los pacientes fueron evaluados en las semanas 2, 4, 6, y 8 (durante la fase de ajuste de la dosis), y después en las semanas 12 y 16 (durante la fase de mantenimiento).

En cada visita se hicieron evaluaciones clínicas y exámenes de sangre según el protocolo. Los pacientes que interrumpieron la terapia por cualquier motivo fueron sometidos a una evaluación de la eficacia en la visita final y no tenían evaluaciones adicionales después de su retiro del estudio. Todos los pacientes que sobrevivieron regresaron para una evaluación de seguimiento de la seguridad a treinta días. Aquellos que completaron el estudio de 16 semanas fueron elegibles para participar en un largo plazo, en el estudio de extensión CHEST-2.

\section{Resultados}

A partir de febrero 2009 hasta febrero de 2012, se sometieron un total de 261 pacientes a la asignación al azar y recibieron al menos una dosis de la medicación de estudio (173 pacientes en el grupo riociguat y 88 en el grupo placebo). No se encontraron diferencias en las características de los pacientes entre los grupos previo al inicio del tratamiento. La mayoría se encontraba en clase funcional WHO II-III y más de la tercera parte tenía el diagnóstico de HPTEC inoperable (más que persistente o recurrente). Un total de 18 pacientes se retiraron del estudio antes de la semana 16. Para diccha semana, $77 \%$ de los pacientes que aún participaban en el estudio tomaban la dosis máxima de riociguat de 2,5 $\mathrm{mg}$ tres veces al día, mientras que en el $10 \%$ (18 pacientes) fue necesario reducirla durante el estudio comparado con el $3 \%$ en el grupo placebo (3 pacientes).

Para evaluar las diferencias entre los grupos durante la prueba de marcha de 6 minutos, se hizo una prueba de hipótesis a dos colas (alfa 0,05 para la diferencia entre tratamientos), en la que se documentó un aumento en la distancia media de 39 metros para el grupo de riociguat, en comparación con una reducción media de 6 metros en el grupo placebo (IC 95\%: 25-67; $\mathrm{p}<0,001)$.

En cuanto a los desenlaces secundarios, la RVP se redujo en 226 dinas $/ \mathrm{seg} / \mathrm{cm}^{-5}$ en el grupo riociguat, en comparación con un aumento de $23 \mathrm{dinas} / \mathrm{seg} / \mathrm{cm}^{-5}$ en el grupo de placebo (IC 95\%: -303 a -190; $p<0,001$ ). Riociguat también se asoció con una mejoría significativa en otras variables hemodinámicas, incluyendo la PAPm y el gasto cardíaco. Los cambios en los niveles de NT-proBNP y la clase funcional fueron estadísticamente significativos y a favor del grupo tratado con riociguat. No hubo diferencia significativa en la 
incidencia de eventos adversos y en el tiempo hasta el empeoramiento clínico entre los grupos de riociguat y placebo $(2 \%$ y $6 \%$, respectivamente, $\mathrm{p}=0,17)$. El índice de disnea de Borg disminuyó en 0,8 puntos en el grupo riociguat y aumentó en 0,2 puntos en el placebo $(p=0,004)$.

Hubo una diferencia nominalmente importante entre los dos grupos en el cambio en la puntuación del cuestionario EQ-5D, pero no en el cambio en la puntuación del cuestionario de vivir con hipertensión pulmonar (LPH).

Los eventos adversos graves relacionados con el tratamiento para el grupo riociguat incluyeron: síncope en tres pacientes ( $2 \%)$, gastritis, insuficiencia renal aguda e hipotensión, en un paciente, respectivamente (1\%).

\section{Conclusión}

En este estudio, riociguat mejoró significativamente la capacidad de ejercicio, determinada por la mejoría en la distancia caminada durante la prueba de marcha de 6 minutos cuando se comparó con placebo en pacientes con HPTEC inoperable, persistente o recurrente. Existió una mejoría significativa en la clase funcional como también en objetivos secundarios como la reducción en los niveles del péptido natriurético (NT-proBNP) y en los valores de la RVP.

\section{Comentario}

La HPTEC constituye el único tipo de hipertensión pulmonar que puede ser aliviada en forma definitiva mediante la trombo-endarterectomía pulmonar. Hasta ahora no existía una alternativa farmacológica efectiva para aquellos con lesiones trombóticas distales o quirúrgicamente no accesibles, para aquellos con alto riesgo quirúrgico dado por otras comorbilidades y para pacientes con hipertensión pulmonar persistente post-operatoria o recurrente. La práctica médica habitual en estos tres escenarios ha sido la utilización de los diferentes agentes anti-proliferativos (bosentán, sildenafil, iloprost, ambrisentán y epoprostenol) aprobados para el tratamiento de la hipertensión arterial pulmonar (HAP) (Grupo $1 \mathrm{OMS}$ ) pero que no lo están en la HPTEC. De ellos, el bosentán es el único que cuenta con un experimento clínico de 157 sujetos con HPTEC (BENEFiT) (1) que tenían cualquiera de las tres condiciones previamente enunciadas y en donde eran aleatorizados a recibir bosentan $125 \mathrm{mg}$ cada 12 horas durante 12 semanas o placebo. El objetivo primario, que consistía en mejorar la distancia durante la prueba de caminata de 6 minutos, al final del periodo de tratamiento no se logró aunque sí hubo una reducción significativa en las resistencias vasculares pulmonares (-24\%). El estudio sugiere que tal vez este desenlace utilizado corrientemente en HAP no debía ser el desenlace primario en esta patología, que, si bien comparte características similares, tiene curso clínico y evolución diferentes.

Riociguat llena este vacío al ser el primer vasodilatador efectivo en la HPTEC al mejorar la capacidad de ejercicio durante la prueba de marcha de 6 minutos. Al estudio se le critica desaprovechar una oportunidad de utilizar un desenlace final con mayor impacto clínico y no uno intermedio como la caminata. El "tiempo hasta el deterioro" (desenlace compuesto que incluye: necesidad de adicionar otro medicamento, deterioro de la clase funcional, hospitalización, necesidad de prostanoide endovenoso, trasplante pulmonar o muerte), fue sugerido desde el $4^{\circ}$ Consenso Mundial de Expertos en Dana Point para el desarrollo de nuevas moléculas en HAP e HPTEC y que en esta ocasión no fue considerado. Riociguat apuesta por lo seguro, desenlaces clásicos primarios (caminata de 6 minutos) y secundarios (RVP) que si bien son importantes, ofrecen poca claridad más allá del impacto clínico. La principal dificultad potencial para este agente es la necesidad de una titulación hasta una dosis máxima según síntomas y cifras de tensión arterial, un obstáculo difícil de sortear en el sistema actual para los pacientes y sus tratantes. Por lo demás, es un estudio bien planteado respecto al diseño y da respuesta a la pregunta de investigación.

\section{Conflicto de intereses}

El Doctor Claudio Villaquirán Torres ha sido asesor y conferencista de Biotoscana, Glaxo SmithKline y Bayer.

\section{Bibliografía}

1. Jaïs X, D'Armini AM, Jansa P, Torbicki A, Delcroix M, Ghofrani HA, et al. Bosentan Effects in iNopErable Forms of chronIc Thromboembolic pulmonary hypertension Study Group. J Am Coll Cardiol. 2008;52(25):2127-34. 